\title{
The Effect of Tau Antisense Oligonucleotides on Neurite Formation of Cultured Cerebellar Macroneurons
}

\author{
Alfredo Caceres, Sonja Potrebic, and Kenneth S. Kosik \\ Department of Neurology (Neuroscience), Harvard Medical School, and Center for Neurologic Diseases, Department of \\ Medicine (Division of Neurology), Brigham and Women's Hospital, Boston, Massachusetts 02115
}

\begin{abstract}
Tau, a microtubule-associated protein (MAP) enriched in axons, may have a role in the generation and maintenance of an axonal morphology. Neurons from embryonic day 15 rat cerebellum in culture elaborate two morphologically distinct neurite populations-one with nontapering, elongated axonlike neurites and the other with tapered dendritelike neurites that branch frequently and are selectively stained with antibodies to MAP2. Tau antisense oligonucleotides were utilized in two ways: (1) continuous application of antisense every $24 \mathrm{hr}$ for variable periods of time or (2) application of antisense that was delayed until neurite differentiation was underway. In both cases, $24 \mathrm{hr}$ after the administration of the antisense, tau protein was not detected immunocytochemically. When the antisense was given continuously directly after plating, the neurites persisted as simple minor outgrowths. When antisense was added $72 \mathrm{hr}$ after plating, axonlike neurites were lost, while the remaining neurites continued to grow and increase in complexity. We concluded that the initial establishment of an elongated axonlike neurite is a prerequisite for further neurite maturation; however, once the axon is established, the remaining neurites are able to grow independently and assume a tapered dendritelike appearance.
\end{abstract}

Axons are collectively defined by morphological, biochemical, and physiological criteria. A key morphological feature of an axon is its nontapering, elongated structure. Because the microtubule system represents a link to structure, it is reasonable to consider how microtubules contribute to the generation of an elongated axonlike structure. A related question is how microtubules contribute to the generation of two morphologically distinct sets of neurites-axons and dendrites. The orientation of the plus and minus ends of microtubules serves to distinguish axons and dendrites (Burton and Paige, 1981; Filliatreau and DiGiamberdino, 1981; Heidemann et al., 1981; Baas et al., 1988). In axons, the plus ends are aligned distally, whereas in dendrites, microtubules have a mixed orientation, particularly in the midportion of the dendrite. Because the two microtubulebased motor molecules, kinesin and dynein, are defined in part

\footnotetext{
Received Sept. 14, 1990; revised Dec. 21, 1990; accepted Jan. 3, 1991.

We are indebted to G. Hall, G. Lee, A. Andreadis, and A. Ferreira for their critical reviews of the manuscript and to R. Maciewicz for use of his Eutectics Neuron Tracing System. The studies were supported by NIH Grants AG06601 and AG06172.

Correspondence should be addressed to Kenneth S. Kosik, M.D., Center for Neurologic Diseases, Brigham and Women's Hospital, 75 Francis Street, Boston, MA 02115 .

Copyright (C) 1991 Society for Neuroscience $0270-6474 / 91 / 111515-09 \$ 03.00 / 0$
}

by the direction that they move organelles in relation to the asymmetry of the microtubule, the contrasting orientations of microtubules within axons and dendrites could give rise to the distinct subcellular components that contribute to the ultrastructural identity of these structures (Black and Baas, 1989).

When neurons from various sites within the developing nervous system are cultured, they elaborate neurites, which may over time approximate some aspects of their in situ morphology. Neurites from several neuronal culture systems, including hippocampus, cerebellum, sympathetic ganglia, and cerebrocortex, develop the morphological characteristics of axons and dendrites (Bartlett and Banker, 1984; Peng et al., 1986; Kosik and Finch, 1987; Ferreira and Caceres, 1989). The axon- and dendritelike structures of cultured hippocampal neurons have been characterized in the most detail. Ultrastructural analyses of these two classes of neurites revealed the presence of ribosomes and postsynaptic elements in dendritelike processes and the absence of ribosomes and presynaptic elements in axonlike structures (Bartlett and Banker, 1984). Tritiated uridine is transported selectively into cultured hippocampal dendrites (Davis et al., 1987).

Two microtubule-associated proteins (MAPs) can also serve as markers of axons or dendrites. The MAP family is a highly diverse group of proteins that can promote microtubule polymerization and codistribute with microtubules in cells. Segregation of the somatodendritic marker MAP2 has been shown in hippocampal (Caceres et al., 1984), cerebellar (Ferreira et al., 1989), cerebrocortical (Kosik and Finch, 1987), and sympathetic cultures (Peng et al., 1986). Tau protein has been shown to segregate to the axon in cerebellar (Ferreira et al., 1989), cerebrocortical (Kosik and Finch, 1987), and sympathetic cultures (Peng et al., 1986). The principal site of synthesis of tau in the brain is in neurons, on a population of mRNAs in the neuronal cell body and within the most proximal portion of the dendrite (Kosik et al., 1989a).

In PC1 2 cells, neurite outgrowth correlates with the induction of tau protein (Drubin et al., 1985) and tau mRNA (Drubin et al., 1988); therefore, a suggested function of tau involves neurite elongation. When infected into the foreign host cell (Sf9 cells from the moth ovary) via a baculovirus, tau induced lengthy process outgrowths (Kosik et al., 1990). Tau may also have a role in the generation of asymmetric neuronal morphologies, because tau antisense exposure in neuronal cell culture specifically inhibits the elaboration of an elongated, nontapering axonlike structure (Caceres and Kosik, 1990).

Shortly after neurons are plated, the minor neurites begin to differentiate, the first into an axon, and then the others into 
dcndrites. A minor ncurite becomes defined as an axon in hippocampal neurons once it has elongated $10 \mu \mathrm{m}$ longer than any other neurite (Goslin and Banker, 1989). Uniformly aligned microtubules are present in minor neurites (Baas et al., 1989) and therefore cannot be used to ascertain the onset of polarity. To identify those molecules important in the early definition of an axon, one strategy has been to localize molecules immunocytochemically within the neurite that is transforming to an elongated axonlike structure. The molecules reported to meet these criteria are GAP43 (Goslin et al., 1988), synapsin, and synaptophysin (Fletcher et al., 1989). However, these molecules do not provide a direct link to the microtubules, the structure required for elongation and possibly for the spatial configuration of an axonlike structure.

To study the interdependence of axonal and dendritic growth, we administered tau antisense oligonucleotides in a sustained manner from the time of plating and in a delayed manner after neurite differentiation was underway. Antisense oligonucleotides can be taken up by cells from the media in a saturable, size-dependent manner consistent with receptor-mediated endocytosis (Loke et al., 1989). We have shown that neurons with a sustained exposure to tau antisense from the time of plating remain locked as an array of minor neurites, while failing to develop the morphologically distinct neurites characteristic of axons or dendrites. On the other hand, the addition of tau antisense oligonucleotides to cultures that have established elongated axonlike structures results in the apparent regression of the most elongated neurite and the continued elaboration of the other neurites.

\section{Materials and Methods}

Cell cultures. Dissociated cultures of cerebellar macroneurons, prepared from embryonic day 15 rat embryos, were dissociated and plated onto polylysine-coated coverslips at a uniform density of $10,000-20,000 / \mathrm{cm}^{2}$ (Ferreira et al., 1989). After $1 \mathrm{hr}$ in serum-containing medium, cells were maintained in D-minimum essential medium/Ham's F12 supplemented with the N2 mixture of Bottenstein and Sato (1979). All the cultures were grown in a $37^{\circ} \mathrm{C}$ incubator with $5 \% \mathrm{CO}_{2}$. Two nonoverlapping tau antisense oligonucleotides designated RT11-14 and RT23 were used at $50 \mu \mathrm{M}$ in all the experiments to show that the effects were specific to the tau sequence. The degree to which these oligonucleotides entered the cells and the response to varying doses were described previously (Caceres and Kosik, 1990). Antisense RT11-14 is the rat tau nucleotide GGTTCAGCCATGCTGCTTCAAAGCC, which corresponds to the inverse complement of nucleotides -14 to +11 (Kosik et al., 1989b). Antisense RT23 is the nucleotide TGATAATCGACAGGAGGCGAGGACA, which corresponds to the inverse complement of nucleotides -15 to -25 (Kosik et al., 1989b). The rat tau sequences selected represent adjacent regions, one that spans the intiator AUG and the other immediately upstream. The sequences selected were not found in the data base in any other known sequence. Adjacent control wells were treated with the same concentration of the corresponding sense-strand oligonucleotide. The oligonucleotides were synthesized on an Applied Biosystems 380B synthesizer, purified over an NAP5 column (Pharmacia), ethanol precipitated, and taken up in medium.

Antisense oligonucleotides were either administered continuously from the time of plating or delayed until after the onset of polarity. When administered continuously, the tau oligonucleotides were added $1 \mathrm{hr}$ after plating, and again at $24-\mathrm{hr}$ intervals to $48 \mathrm{hr}$, and fixed for immunocytochemistry at $72 \mathrm{hr}$. When administered in a delayed fashion, a single dose of tau antisense was added after the cells were in culture for $72 \mathrm{hr}$ and then fixed $24 \mathrm{hr}$ later. One additional protocol was utilized in which antisense oligonucleotides were added with a delay of only 24 $\mathrm{hr}$, and in some cases, a second dose was administered at $48 \mathrm{hr}$. These cultures were fixed $24 \mathrm{hr}$ after the last dose of antisense. For each experiment, several plates were not fixed at the determined time point, but were allowed to recover from the effects of the antisense.

Immunocytochemistry. The tau antibodies used were 5E2 (Kosik et al., 1988b) and tau 1 (Binder et al., 1985); both monoclonals were used at $1: 20$ and 1:100, respectively. The MAP2 antibodies were 5F9, a monoclonal (Kosik et al., 1988a) used at 1:100, and R4, a MAP2 polyclonal (Kosik and Finch, 1987) used at 1:500. Tubulin was labeled with TuJl to total tubulin (Geisert and Frankfurter, 1989). Tyrosinated tubulin and MAPla antibodies were obtained from Sigma Chemical Co. (St. Louis, MO). The secondary antibodies used were the mouse extravidin staining kit (Sigma) with diaminobenzidine as the chromagen, rabbit anti-mouse IgG biotin conjugate (Sigma), goat anti-mouse IgG rhodamine conjugate (Calbiochem), extravidin fluorescein isothiocyanate (FITC) conjugate (Sigma), and goat anti-rabbit IgG rhobdamine conjugate (Sigma).

For immunocytochemistry, cells were fixed with either cold methanol $\left(-20^{\circ} \mathrm{C}\right)$ or $4 \%$ paraformaldehyde in PBS with $0.12 \mathrm{M}$ sucrose at $37^{\circ} \mathrm{C}$. Paraformaldehyde-fixed cells wcre permcabilized in $0.2 \%$ Triton X-100 in PBS for 5 min. For double-labeling immunofluorescence, the cells were incubated with polyclonal and monoclonal antibodies simultaneously, washed, and incubated with the secondary antibodies goat antirabbit TRITC conjugate, rabbit anti-mouse $\operatorname{IgG}$ biotin conjugate, and extravidin FITC conjugate together. In all experiments, the coverslips were preincubated with $5 \%$ bovine serum albumin. Photomicrographs were taken on an Carl Zeiss microscope equipped with epifluorescence illumination through $40 \times$ or $63 \times$ objectives using TMAX 100 or 400 ASA film (Eastman Kodak Co.).

Analysis of cultures. Developing cerebellar neurons were staged according to Ferreira et al. (1989) and are illustrated in Figure 1. Stage I cells are either rounded or have a lamellipodial veil. Stage II cells have a fairly symmetric array of short, unbranched neurites (Fig. $1 A$ ). The transition from stage II to stage III represents the acquisition of morphological polarity in that a single neurite begins to elongate (Fig. $1 B$ ). At stage III, one neurite has a relatively uniform diameter over most of its length and thus has the appearance of an axon (Fig. 1C). Stage IV occurs when the minor neurites develop a tapered, highly branched appearance, similar to that of dendrites (Fig. $1 D, E$ ). Analogous stages of maturation have been described for cultured hippocampal neurons (Dotti et al., 1988).

Some cultures were drawn using a $63 \times$ ocular on the Eutectics Neuron Tracing System (Johnson and Capowski, 1983) and analyzed according to its software. Camera lucida drawings were made, including a set with a $100 \times$ oil-immersion objective to determine neurite caliber at the tip. All drawings were made from slides stained with the tubulin or MAP2 antibody. Bright-field drawings were checked by phase-contrast microscopy to be sure all ncurites werc drawn.

\section{Results}

\section{Effect of prolonged exposure to tau antisense}

Tau antisense oligonucleotides were added to the media of cerebellar macroneurons in culture from the time of plating until $48 \mathrm{hr}$ to determine the effects of sustained antisense exposure on morphological development. The abolition of detectable tau protein in antisense-treated cultures was demonstrated previously by dot immunobinding (Caceres and Kosik, 1990) and is shown here by immunocytochemistry (Figs. 2, 3). Staining by two tau monoclonal antibodies (5E2 and tau 1) revealed backround levels of reaction product over the entire treated culture. Both antisense oligonucleotides (RT11-14 and RT23) were equally effective in inhibiting tau labeling. In all of the paradigms, the sense- and antisense-treated cultures synthesized other proteins as determined by their reactivity with tubulin, MAPla, and MAP2 antibodies (Figs. 2, 3).With a sustained dose of antisense from the time of plating, cultured neurons extended initial cxploratory neurites and maintained a symmetric array of minor neurites (Fig. 2). Control sense-treated cultures, on the other hand, not only extended initial neurites, but also elongated a single neurite within the first $24 \mathrm{hr}$ that developed the appearance of an axon. Neurons could be kept locked at this stage by administration of tau antisense every 24 hr. At $72 \mathrm{hr}$ in culture, cells treated according to the sustained 

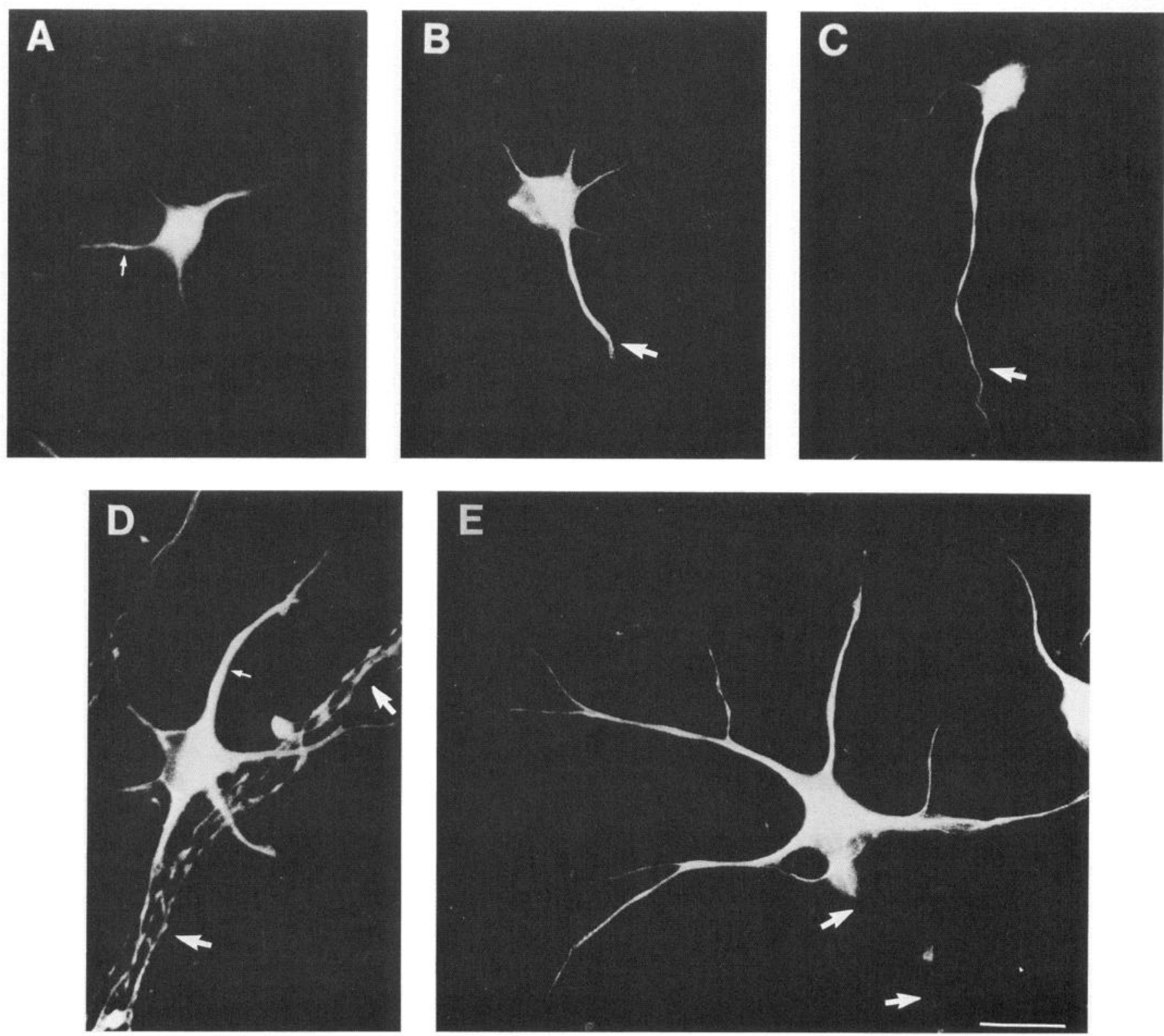

Figure 1. Development of cerebellar macroneurons labeled with tau monoclonal antibody tau $1(A-C)$ or MAP2 (R4) antibody $(D, E)$. $A$, Stage II cell with minor neurites (arrow) after $12 \mathrm{hr}$ in culture. $B$, Transition to stage III after $24 \mathrm{hr}$ in culture; the arrow shows an elongating neurite. $C$, Stage III cell with a single elongated neurite (arrow) and very-fine-caliber remaining minor neurites after $24 \mathrm{hr}$ in culture. At this stage, the remaining minor neurites become thinner. $D$, Stage IV neuron after $4 \mathrm{~d}$ in culture, stained with MAP2 antibody, at which time the caliber of the minor neurites increases and many neurites appear tapered (small arrows); the large arrows point to a bundle of axons, which at this time point are still reactive with the MAP2 antibody. $E$, Seven days after plating, only the dendritelike neurites are labeled with the MAP2 antibody.The arrows point to the trajectory of an axon apparent only by phase contrast. Scale bar, $10 \mu \mathrm{m}$.

exposure protocol markedly contrasted with control cells, which had elongated axonlike structures (cf. Figs. 1, 2). The effect of antisense treatment was very different from a complete interference with protein synthesis as determined by the administration of cycloheximide at comparable times. This treatment resulted in many nonviable neurons and the complete inhibition of any neurite outgrowth (data not shown).

When the daily administration of antisense was discontinued after $72 \mathrm{hr}$ and the cells allowed to recover, they acquired polarity and continued to differentiate their neurites within a time frame identical to cells plated at the time antisense was discontinued (Fig. 2D,E). Cells fixed at $120 \mathrm{hr}$ (Fig. 2D) and at 144 hr (Fig. 2E) after plating and antisense treatment first formed a single elongated neurite (Fig. $2 D$ ), followed by the development of branched and tapered neurites (Fig. $2 E$ ). Release from the phenotypic effects of tau antisense appeared to occur within $24-48 \mathrm{hr}$ of the cessation of antisense administration. This time period, which must approximate the half-life of the antisense, was the basis for selecting a $24-\mathrm{hr}$ interval between doses. The cells were able to recover from the sustained exposure paradigm and resume development from the point at which they were arrested. Antisense treatment did not irreversibly impair the viability or the ability of the neurons to differentiate. However, over the period observed, neurite growth failed to catch up with the controls and maintained a lag commensurate with the time in antisense. 



Figure 2. Continuous exposure to tau antisense given 1, 24, and $48 \mathrm{hr}$ after plating and fixed at $72 \mathrm{hr}$. $A$ and $B$ are double-labeled neurons stained with MAP2 antibody R4 $(A)$ and tau antibody tau $1(B)$. Only trace tau immunoreactivity is detectable. $C$ is an identically treated plate of cells stained with tyrosinated tubulin antibody. The appearance of these cells is similar to the stage II cell shown in Figure $1 A$. $D$ and $E$ are recovering cells stained with tau 1, $2(D)$ and $3(E)$ d following the cessation of antisense administration. Scale bar, $15 \mu \mathrm{m}$.

\section{Effect of tau antisense administration after the onset of polarity}

Tau antisense oligonucleotides were administered after the onset of polarity to determine their effect on cells that had already formed an axonlike neurite. Preliminary to determining the effects of tau antisense on neurites, we first established that two morphologically distinguishable populations of neurites were present in this culture system. Because tapering of neurites is considered a close correlate of dendritic identity (Baas et al., 1989), the degree of tapering was quantitated among primary branches and secondary branches that did not undergo further branching in cerebellar macroneurons cultured for $7 \mathrm{~d}$ (Fig. 4). Neurites fell into two populations - those that tapered and those that did not. For nearly all cells, a single nontapered neurite could be identified. Measurements of the dendritic diameter just proximal to any terminal expansion gave a range of diameters from 0.8 to $1.2 \mu \mathrm{m}$. The terminal diameter of axonlike neurites was $0.5-0.8 \mu \mathrm{m}$. These values may also distinguish axons and dendrites (Banker and Waxman, 1988).

At $72 \mathrm{hr}$ after plating, cerebellar cultures were treated with tau antisense for $24 \mathrm{hr}$ and then fixed for immunocytochemistry (Fig. 3). Tau immunoreactivity was abolished; however, tubulin and MAP2 immunoreactivity were identical to the controls. 

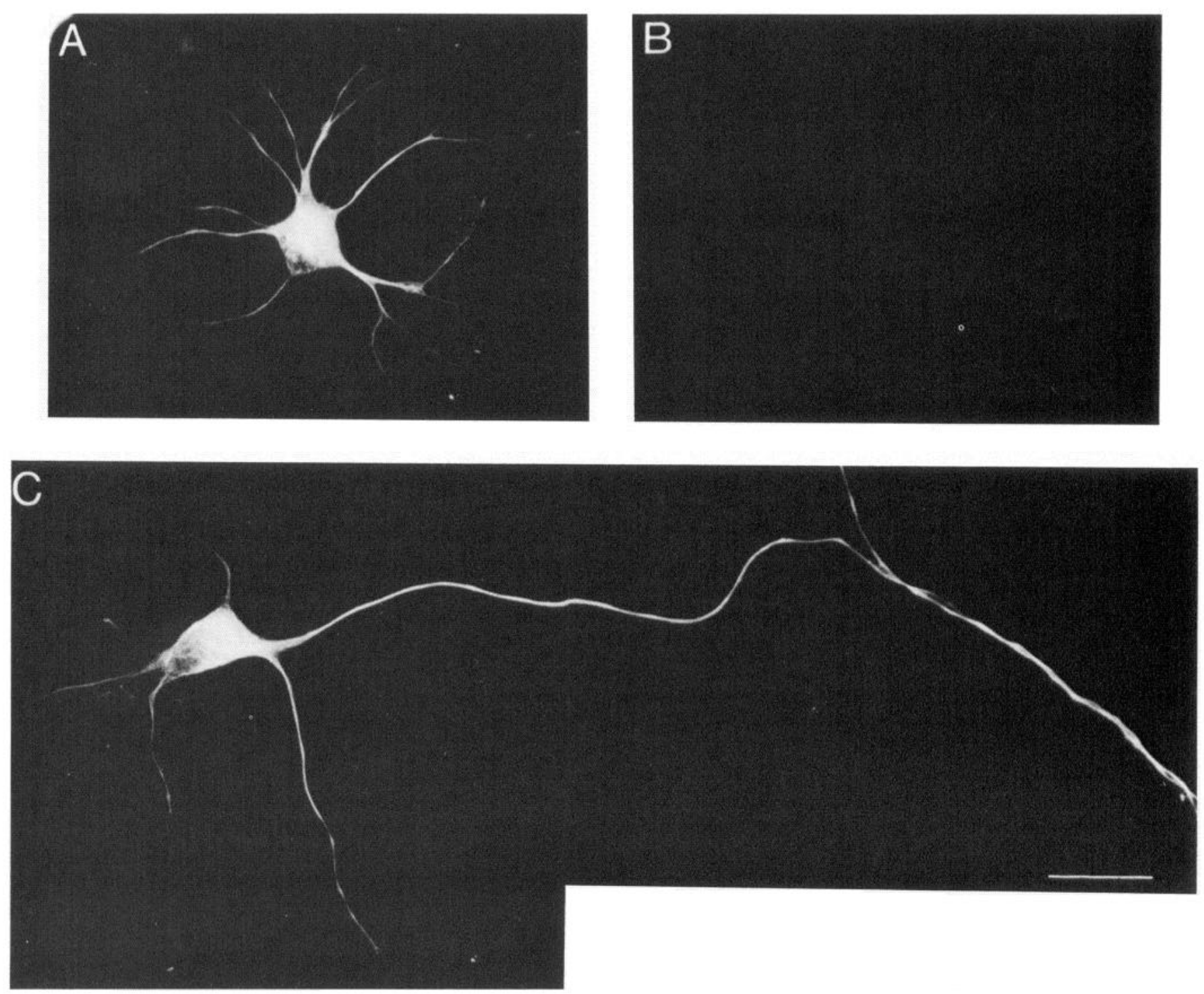

Figure 3. Delayed exposure of cerebellar macroneurons to tau oligonucleotides given as a single dose $72 \mathrm{hr}$ after plating. Cells were fixed $24 \mathrm{hr}$ later and double labeled with tau antibody 5E2 (1:20) and MAP2 polyclonal antibody R4 (1:500). $A$ and $B$ represent the tau antisense, and $C$, the sense control. Tau immunoreactivity is demonstrated in $B$ and $C$, and MAP2 immunoreactivity, in $A$. Scale bar, $10 \mu \mathrm{m}$.

Likewise, MAPla immunoreactivity was identical to controls (data not shown). Double labeling of treated cells with tau and MAP2 antibodies demonstrated that cells with intense MAP2 immunoreactivity were not stained by the tau antibody under antisense conditions (Fig. $3 A, B$ ). The most dramatic difference between the appearance of the antisense and control cells was the nearly complete lack of neurites that resembled axons. Because tapering distinguished neurite populations, a plot of thickness versus distance from the cell body for primary and secondary neurites was used to demonstrate that the remaining population of neurites after antisense treatment had a dendritelike morphology (Fig. 4). In untreated cultures, there was a highly significant inverse correlation for the population of dendritelike neurites; for the population of neurites arising from the cell body with an axonlike appearance, the caliber did not change as a function of distance from the cell body beyond the initial $5 \mu \mathrm{m}$. Some axons arose from the proximal portion of dendritelike processes, in which there was occasionally some ta- pering over the first $10 \mu \mathrm{m}$. Thickness versus distance measurements of the neurites from cultures treated with delayed administration of antisense had a correlation coefficient identical to that of the dendritelike population of neurites from untreated cultures (Fig. 4). When these cells remained in culture beyond the 24 -hr period between antisense administration and fixation, they recovered normally.

Thirty cells treated with delayed administration of oligonucleotides were labeled with the tubulin antibody, and randomly selected fields of the sense- and antisense-treated cultures were drawn with the Eutectics Neuron Tracing System (Johnson and Capowski, 1983). Representative cells are shown in Figure 5. While an axonlike neurite was identifiable in nearly all of the sense-treated neurons, only 6 of 30 antisense-treated cells had a similar axonlike neurite that could be distinguished from the other neurites on the basis of length and lack of tapering. Although these six cells appeared to have escaped the morphological effects of the antisense, the lack of tau immunoreactivity 

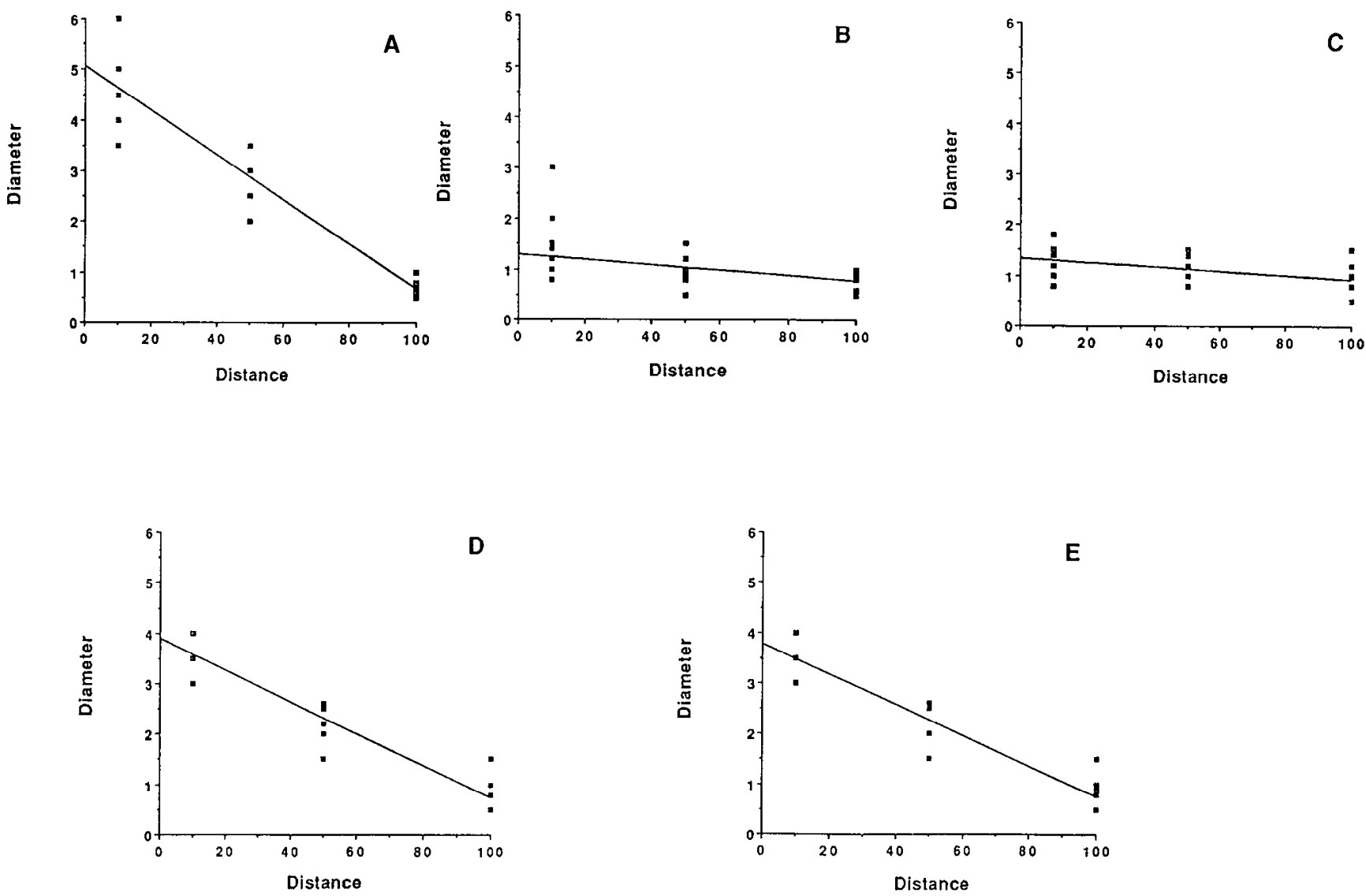

Figure 4. Relationship of the neurite diameter to the distance from the cell body for dendritelike processes after $7 \mathrm{~d}$ in culture $(A)$, axonlike processes after $7 \mathrm{~d}$ in culture $(B)$, minor neurites after $1 \mathrm{~d}$ in culture $(C)$, neurites after $4 \mathrm{~d}$ in culture $(D)$, and neurites treated with tau antisense given at $72 \mathrm{hr}$ after plating and fixed $24 \mathrm{hr}$ later $(E)$. Diameters are in microns, and the distance from the cell body is standardized for the variable lengths of cultures $A-E$ by setting the neurite tip at 100 and measuring the diameter at three points that represent percentages of the total length. The correlation coefficients $(r)$ with length are $A, 0.92 ; B, 0.24 ; C, 0.24 ; D, 0.87 ;$ and $E, 0.92$. The analysis utilized primary neurites that did not undergo further branching. Twenty cells with an average of 2.5 neurites per cell were drawn for each graph.

was homogeneous over the entire culture. Ten antisense-treated neurons had a single neurite with unusual morphological features, such as multiple branches emanating from a common origin or unusual branch angles. The loss of a single elongated or axonlike neurite is quantitated in Figure 6, which shows that the length of the longest neurite per cell was significantly greater under the control conditions and that antisense-treated neurons had increased numbers of shorter neurites. Comparison of all the neurites from the antisense-treated population revealed a significantly shorter total summed neurite length, thicker primary and secondary neurites, and neuritic volumes equal to the sense-treated cells (Table 1).

Neurite complexity was analyzed by the probability of branching from each segment (Caceres and Steward, 1983). The average number of branches per cell was greater in the antisensetreated cultures (antisense, 17.6 \pm 10.8 ; sense, $11.5 \pm 7.8 ; p<$ 0.01 ). There were $3.93 \pm 0.25$ second-order segments in the sense cultures and $5.86 \pm 0.10$ in the antisense cultures $(p<$ 0.001 ). There were $1.93 \pm 0.10$ third-order branches in the sense cultures and $3.44 \pm 0.16$ in the antisense cultures $(p<0.001)$. The likelihood of second-order segments branching further was 0.32 for the antisense and 0.22 for the sense. The likelihood of third-order segments branching further was 0.28 for the antisense and 0.15 for the sense. The number of first-order segments arising from the soma did not differ in the two conditions (sense,
$4.06 \pm 1.29 ;$ antisense, $4.33 \pm 1.83$ ). Therefore, the increase in branch complexity is due to higher-order sprouting. Because an axon could not be definitively identified in many of the antisense-treated cells, these calculations were done in several ways: (1) inclusion of all neurites without assumptions regarding dendritic or axonal identity; (2) exclusion of the axon from the sense-treated cultures; (3) exclusion of the axon from the sensetreated cultures and the systematic exclusion of a single neurite from each of the antisense-treated cells; and (4) exclusion of any axonal-appcaring structure from both groups. All methods of calculation gave significantly increased branching in the antisense cultures; the most conservative values (calculation 3 ) are presented.

The presence of tapered neurites that continued to increase in complexity while exposed to tau antisense suggested that this population of neurites was resistant to the effects of the antisense. Together the results from the two protocols (summarized in Fig. 7) suggested that growth of highly branched tapered neurites could occur in the absence of an elongated axonlikc neurite, but only after some degree of neurite differentiation had already begun to occur. Cells with early and sustained exposure to antisense did not develop complex branched neurites. An additional intermediate time point of antisense administration was tried to demonstrate that the response to antisense at $72 \mathrm{hr}$ was developmentally distinct from that observed with contin- 




A

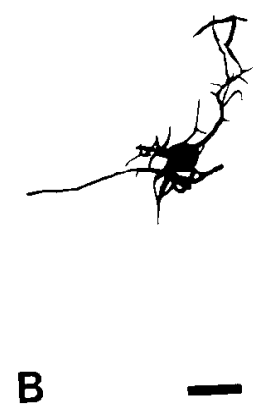

Figure 5. Representative cells from sense- $(A)$ and antisense-treated $(B)$ cultures, stained with anti-tubulin (TuJ1), and traced with the Eutectics system under $63 \times$ oil immersion lens directly from the microscope stage. Thickness values were entered as the cells were traced. Scale bar, $15 \mu \mathrm{m}$.

uous application from the time of plating. Neurons were treated with antisense oligonucleotides after $24 \mathrm{hr}$ in culture, a time when the population of neurites appears to consist only of simple minor neurites and a single elongated neurite on many cclls. Some cultures were given a second dose of tau antisense at 48 hr after plating. Fixation $24 \mathrm{hr}$ after the last dose of antisense revealed cells identical to those treated with continuous application of antisense from the time of plating. Therefore, cerebellar neurons must develop for at least $24 \mathrm{hr}$ in culture before their minor neurites are able to maintain sustained growth in the presence of tau antisense.

Finally, when the application of tau antisense was delayed until $96 \mathrm{hr}$ after plating and the cells fixed $24 \mathrm{hr}$ later, there was no detectable effect on tau immunoreactivity or on the morphology. Whether continuous application of tau antisense beginning at $96 \mathrm{hr}$ could affect neurite growth was not tested, but might be effective if the half-life of tau has increased with neuronal maturity.

\section{Discussion}

The analysis of 10-d-old cerebellar neurons revealed two populations of neurites - those that tapered and branched at relatively short distances, and those that did not taper and had an elongated appearance. As neurites are elaborated from cerebellar neurons, they follow a highly reproducible pattern of maturation (Fig. 1). Within the first $12 \mathrm{hr}$ of plating, the neurons elaborate a symmetric array of minor neurites that are not lapered (Fig. 1A). By $24 \mathrm{hr}$, most of the cells have elaborated an axonlike structure, while the remaining minor neurites have not appre-

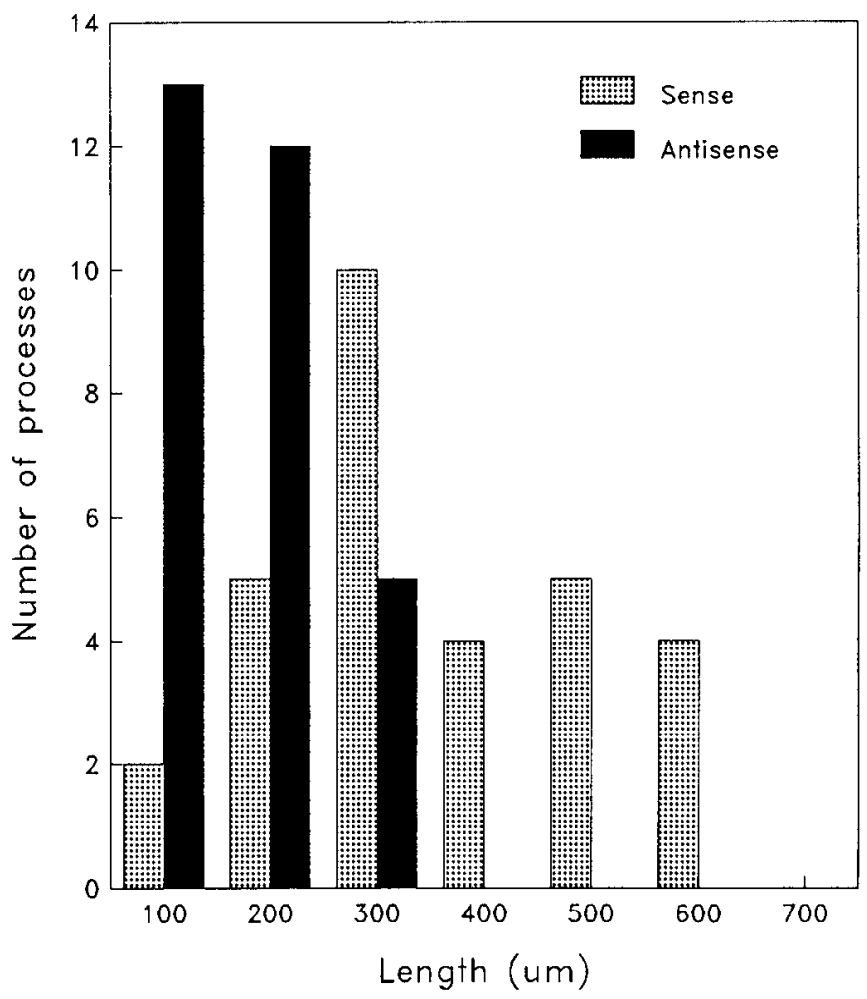

Figure 6. The left shift to shorter neurites is shown in the antisensetreated population. The mean value for the longest sense-treated neurite is $302.0 \mu \mathrm{m}$, with a range of $45.7-551.3 \mu \mathrm{m}$. The mean value for the longest antisense-treated neurite is 130.5 , with a range of $27.1-298.3$ $\mu \mathrm{m}$. This difference was highly significant $(p<0.0003)$ using the MannWhitney $U$ test for the nonparametric distribution of the histogram.

ciably changed. At $4 \mathrm{~d}$ in culture, the minor neurites show tapering and the complex branching pattern typical of dendrites (Fig. 1D).

The continuous exposure to tau antisense permitted the hypothesis that the prior development of an elongated axonlike neurite was necessary for the conversion of minor neurites into dendrites. Once the cell passed the stage of axonal formation, a distinct population of neurites emerged that resembled dendrites. With application of the antisense delayed until $72 \mathrm{hr}$, these dendritelike neurites were capable of continued growth in the absence of an identifiable axon. Therefore, the transition from minor neurites to dendritelike processes requires a chronologically prior stage of axonal initiation. Because this observa-

Table 1. Effects of tau antisense on neuronal shape parameters

\begin{tabular}{lllllc} 
& $\begin{array}{l}\text { Total } \\
\text { length }(\mu \mathrm{m})\end{array}$ & $\begin{array}{l}\text { Total } \\
\text { volume }\left(\mu \mathrm{m}^{3}\right)\end{array}$ & $\begin{array}{l}\text { Thickness/ } \\
1^{\circ} \text { segment }\end{array}$ & $\begin{array}{l}\text { Thickness/ } \\
2^{\circ} \text { segment }\end{array}$ & Soma area $\left(\mu \mathrm{m}^{2}\right)$ \\
\hline Sense & $\begin{array}{l}420.7 \\
(75.6-691.8)\end{array}$ & $\begin{array}{l}167.9 \\
(27.5-482.3)\end{array}$ & $1.1 \pm 0.07$ & $0.5 \pm 0.05$ & $105.2 \pm 4.11$ \\
Antisense & $\begin{array}{l}252.6 \\
(63.8-439.2)\end{array}$ & $\begin{array}{l}188.5 \\
(16.7-737.8)\end{array}$ & $1.4 \pm 0.11$ & $0.7 \pm 0.05$ & $106.8 \pm 4.87$ \\
Significance & $p<0.005$ & $p>0.1$ & $p<0.05$ & $p<0.01$ & $p>0.2$ \\
\hline
\end{tabular}

The parameters listed were compared for cultures treated with either sense or antisense oligonucleotides added at $72 \mathrm{hr}$ after plating and fixed $24 \mathrm{hr}$ later. For the total length and total volume, the significance was determined by the MannWhitney $U$ test because the distribution of values was skewed. For the remaining determinations, a two-tailed Student's $t$ test was used. All values were generated with $n=30$, and errors refer to SEM. The numbers in parentheses are ranges. 
Figure 7. Camera lucida drawings of anti-tubulin-labeled neurons treated with either continuous or delayed exposure to tau antisense oligonucleotides for the number of days in culture indicated along the top. Row $A$ cells were continuously exposed to tau antisense from the time of plating (see Materials and Methods). Row $B$ cells received a singlc dosc of tau antisense $72 \mathrm{hr}$ after plating and were fixed $24 \mathrm{hr}$ later. At 3 $\mathrm{d}$ with daily exposure to antisense since the time of plating, only short minor neurites are evident, whereas untreated cells $($ row $B$ ) at $3 \mathrm{~d}$ show an elongated process. With the cessation of daily antisense treatment, the cells begin to show recovery at $4 \mathrm{~d}$, whereas delayed antisense administration results in the loss of the axonlike neurite and continued growth of the other neurites. By $5 \mathrm{~d}$, cells that were initially exposed to daily antisense administration continue to recover and look similar to untreated cells at day 3 . With a single dose of antisense at day 3 , recovery of the axon by day 5 is apparent. Arrows correspond to neurites that extend beyond the figure.
A
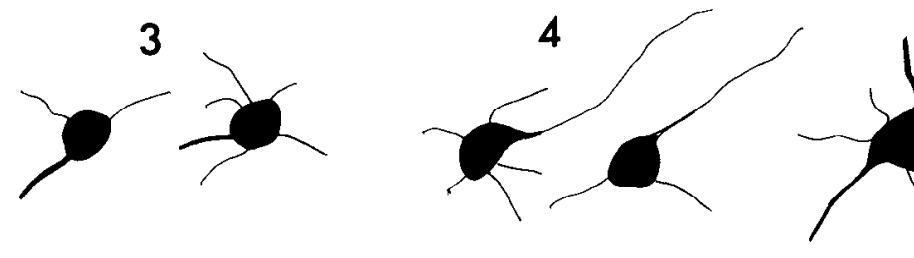

5

B

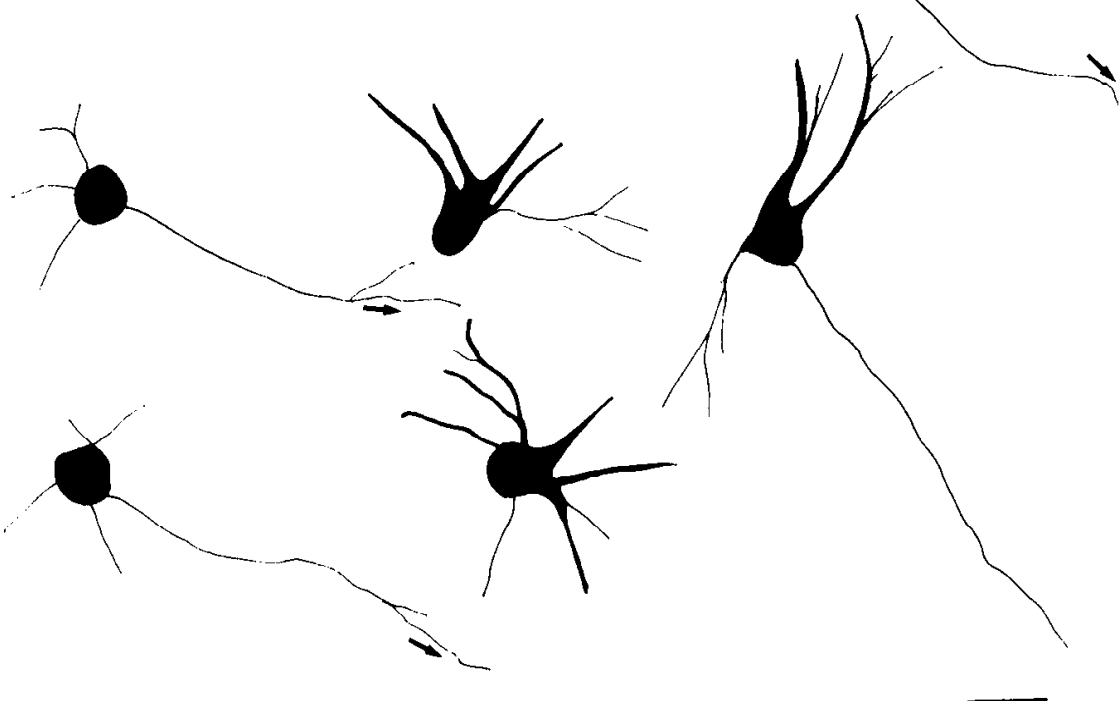

tion suggested a developmental threshold, an additional intermediate time point of antisense application was studied. Oligonucleotides given at $24 \mathrm{hr}$ in culture, a time when polarity is just becoming evident, resulted in morphologies indistinguishable from continuous antisense application from the time of plating. Thus, at $24 \mathrm{hr}$, neurites still require tau synthesis for any differentiation beyond minor neurites. At $72 \mathrm{hr}$, only the elongation of the axonlike neurite is dependent upon continued tau synthesis.

The presence of neurites with only minimal axonal features when antisense was administered at $72 \mathrm{hr}$ suggested neurite regression and a role for tau in sustaining an axon. Dendritic branching complexity has been described as a function of plating density (Banker and Waxman, 1988); however, in this study, under conditions of decreased cell-cell contact due to the loss of the long axon, branching complexity was increased in comparison to controls. In many systems, particularly in vivo systems, when a neuron is deprived of its axon, sprouting occurs. This response usually occurs from the axon stump, but can occur ectopically from dendritic tips after a close axotomy (Hall et al., 1989). It is possible that the enhanced branching complexity after delayed tau antisense administration represented a reactive response to the loss of an axonlike process.

How neurites gain distinct identities as axons and dendrites must cntail a cascade of molecular events that lead to a mature morphology. One aspect of axonal identity is the distinct morphology attained by a single elongated, nontapering structure among a population of neurites. While tau protein probably has a role in the determination of the axon's unique morphology (Caceres and Kosik, 1990), neuronal polarity does not arise from the selective segregation of tau protein to a single neurite. Tau is present in all neurites, including minor neurites at the time of axonal formation. Therefore, a mechanism must exist to inhibit other minor neurites from becoming axons. In hippocampal ncurons, the transection of an axon close to the cell body at early time points releases the inhibition on elongation and permits the conversion of one of the other early neurites to an axon (Goslin and Banker, 1989). To perform a unique role within only one of the neurites, either tau is distinct within a single neurite undergoing elongation, or, as an effector molecule, it acts differentially upon a substrate that is distinct within the incipient axon. Although tau does undergo complex developmentally regulated splicing events (Kosik et al., 1989b), there is no change in the electrophoretic migration of the tau isoforms during the time course of these experiments (Ferreira et al., 1989). A more likely neurite-specific alteration of tau is in its relationship to the microtubule. The binding of tau to microtubules selectively within a single neurite may occur stochastically and serve as a critical step in the formation of the axon. Once tau binds, it may engender cooperative phenomena that enhance additional binding and lead to neurite elongation. Dendritic differentiation can proceed in the absence of tau, once neurons have entered stage III. The MAP2 molecule, with its ability to displace tau from microtubules (Sandoval and Vandekerckhove, 1981), represents a good candidate for a complementary role in dendritic differentiation.

When the 72-hr cultures were fixed after only $16 \mathrm{hr}$ in antisense, considerable tau immunoreactivity remained. Because a 24-hr exposure to the antisense resulted in clear effects on morphology and tau immunoreactivity, the briefer 16-hr interval may represent insufficient time for the turnover of the tau protein pool synthesized before the administration of the antisense. Fixation after $48 \mathrm{hr}$ in the antisense revealed the reappearance of tau immunoreactivity, probably due to exhaustion of the antisense oligonucleotide. When tau antisense was added after $96 \mathrm{hr}$ in culture and analyzed $24 \mathrm{hr}$ later, there was only minimal 
loss of the axonlike neurite. The failure to see a decrement in tau protein after $24 \mathrm{hr}$, when the antisense was added at $96 \mathrm{hr}$ rather than $72 \mathrm{hr}$ in culture, could be explained by a lengthening of the tau protein half-life between these time points. The observed decrement in tau mRNA levels with maturity (Mangin et al., 1989) is consistent with a concomitant lengthening of its half-life. Tau could become less vulnerable to proteolysis by binding to microtubules and proteolytically eliminated when free of microtubules (Okabe and Hirokawa, 1989). Thus, binding of tau to microtubules may represent a critical step in the transformation of an initial exploratory neurite to an axon. Indirect support for the importance of microtubule stability in the elaboration of an axonlike structure stems from the finding that, in cultured, cerebellar neurons, microtubules are first acetylated in the axon (Ferreira and Caceres, 1989). Furthermore, the addition of tau antisense just after plating results in the failure to detect acetylated microtubules, despite the persistence of tubulin reactivity (A. Caceres and $\mathrm{K}$. S. Kosik, unpublished observations). Because the phosphorylation state of tau is known to affect its binding to microtubules (Lindwall and Cole, 1984), a balance between cellular kinases and phosphatases may regulate tau binding to microtubules and thus operate in the generation of polarity.

\section{References}

Baas PW, Black MM, Banker GA (1989) Changes in microtubule polarity orientation during the development of hippocampal neurons in culture. J Cell Biol 109:3085-3094.

Baas PW, Deitch JS, Black MM, Banker GA (1988) Polarity orientation of microtubules in hippocampal neurons: uniformity in the axon and nonuniformity in the dendrite. Proc Natl Acad Sci USA 85:8335-8339.

Banker GA, Waxman AB (1988) Hippocampal neurons generate natural shapes in cell culture. In: Intrinsic determinants of neuronal form and function (Lasek RJ, Black MM, eds), pp 61-82. New York: Liss.

Bartlett WP, Banker GA (1984) An electron microscopic study of the development of axons and dendrites by hippocampal neurons in culture. J Neurosci 4:1944-1953.

Binder LI, Frankfurter A, Rebhun LI (1985) The distribution of tau in the mammalian central nervous system. J Cell Biol 101:13711385 .

Black MM, Baas PW (1989) The basis of polarity in neurons. Trends Neurosci 12:211-214.

Bottenstein JE, Sato GH (1979) Growth of a rat neuroblastoma cell line in serum-free supplemented media. Proc Natl Acad Sci USA 76: 514-517.

Burton PR, Paige JL (1981) Polarity of axoplasmic microtubules in the olfactory nerve of the frog. Proc Natl Acad Sci USA 78:32693273.

Caceres A, Kosik KS (1990) Inhibition of neurite polarity by tau antisense oligonucleotides in primary cerebellar neurons. Nature 343: 461-463.

Caceres A, Steward O (1983) Dendritic reorganization in the denervated dentate gyrus of the rat following entorhinal cortical lesions: a Golgi and electron microscopic study. J Comp Neurol 214:387-403.

Caceres A, Banker GA, Steward O, Binder L, Payne M (1984) MAP2 is localized to the dendrites of hippocampal neurons which develop in culture. Dev Brain Res 13:314-318.

Davis L, Banker G, Steward O (1987) Selective dendritic transport of RNA in hippocampal neurons in culture. Nature 330:477-479.

Dotti CG, Sullivan CA, Banker GA (1988) The establishment of polarity by hippocampal neurons in culture. J Neurosci 8:1454-1468.

Drubin DG, Feinstein SC, Shooter EM, Kirschner MW (1985) Nerve growth factor-induced neurite outgrowth in PC12 cells involves the coordinate induction of microtubule assembly and assembly promoting factors. J Cell Biol 101:1799-1807.
Drubin DG, Kobayashi S, Kellogg D, Kirschner MW (1988) Regulation of microtubule protein levels during cellular morphogenesis in nerve growth factor-treated PC12 cells. J Cell Biol 106:1583-1591.

Ferreira A, Caceres A (1989) The expression of acetylated microtubules during axonal and dendritic growth in cerebellar macroneurons which develop in vitro. Dev Brain Res 49:207-213.

Ferreira A, Busiglio J, Caceres A (1989) Microtubule formation and neurite growth in cerebellar macroneurons which develop in vitro: evidence for the involvement of the microtubule-associated proteins MAP-1a, HMW-MAP2, and tau. Dev Brain Res 49:213-228.

Filliatreau G, DiGiamberdino L (1981) Microtubule polarity in myelinated axons as studied after decoration with tubulin. Biol Cell 42: 69-72.

Fletcher TL, De Camilli P, Banker GA (1989) Synaptogenesis in hippocampal cultures: axons and dendrites become competent to form synapses at different stages of development. Soc Neurosci Abstr 15: 1388.

Geisert EE Jr, Frankfurter A (1989) The neuronal response to injury as visualized by immunostaining of class III $\beta$-tubulin in the rat. Neurosci Lett 102:137-141.

Goslin K, Banker G (1989) Experimental observations on the development of polarity by hippocampal neurons in culture. J Cell Biol 108:1507-1516.

Goslin K, Schreyer DJ, Skene JHP, Banker G (1988) Development of neuronal polarity: GAP-43 distinguishes axonal from dendritic growth cones. Nature 336:672-674.

Hall GF, Poulos A, Cohen MJ (1989) Sprouts emerging from the dendrites of axotomized lamprey central neurons have axonlike ultrastructure. J Neurosci 9:588-599.

Hcidcmann SR, Landers JM, Hamborg MA (1981) Polarity orientation of axonal microtubules. J Cell Biol 91:661-665.

Johnson EM, Capowski JJ (1983) A system for the three-dimensional reconstruction of biological structures. Comput Biomed Res 16:79.

Kosik KS, Finch EA (1987) MAP2 and tau segregate into axonal and dendritic domains after the elaboration of morphologically distinct neurites: an immunocytochemical study of cultured rat cerebrum. J Neurosci 7:3142-3153.

Kosik KS, Orecchio LD, Bakalis S, Duffy L, Neve RL (1988a) Partial sequence of MAP2 in the region of a shared epitope with Alzheimer neurofibrillary tangles. J Neurochem 51:587-598.

Kosik KS, Orecchio LD, Binder L, Trojanowski JQ, Lee V, Lee G (1988b) Epitopes that span the tau molecule are shared with neurofibrillary tangles. Neuron 1:817-825.

Kosik KS, Crandall JE, Mufson E, Neve RL (1989a) Tau in situ hybridization in normal and Alzheimer brain: a predominant localization in the neuronal somatodendritic compartment. Ann Neurol $26: 352-361$.

Kosik KS, Orecchio LD, Bakalis S, Neve RL (1989b) Developmentally regulated expression of specific tau sequences. Neuron 2:1389-1397.

Kosik KS, Caceres A, Pardee J, Cohen-Gould L, Knops J, McConlogue L (1990) A role for tau in neural morphogenesis. J Cell Biol 111: $436 \mathrm{a}$.

Lindwall G, Cole RD (1984) Phosphorylation affects the ability of tau protein to promote microtubule assembly. J Biol Chem 259:53015305.

Loke SL, Stein CA, Zhang XH, Mori K, Nakanishi M, Subasinghe C, Cohen JS, Neckers LM (1989) Characterization of oligonucleotide transport into living cells. Proc Natl Acad Sci USA 86:3474-3478.

Mangin G, Couchie D, Charriere-Bertrand C, Nunez J (1989) Timing of expression of $\tau$ and its encoding mRNAs in the developing cerebral neocortex and cerebellum of the mouse. J Neurochem 53:45-50.

Okabe S, Hirokawa N (1989) Rapid turnover of microtubule-associated protein MAP2 in the axon revealed by microinjection of biotinylated MAP2 into cultured neurons. Proc Natl Acad Sci USA 86: $4127-4131$.

Peng A, Binder LI, Black MM (1986) Biochemical and immunological analyses of cytoskeletal domains of neurons. J Cell Biol 102:252-262.

Sandoval IV, Vandekerckhove JS (1981) A comparative study of the in vitro polymerization of tubulin in the presence of microtubuleassociated proteins MAP2 and tau. J Biol Chem 256:8795-8800. 